\title{
Determination of heat flux intensity distribution and laser absorption rate of AISI D2 tool steel
}

\begin{abstract}
The prediction of fluctuated temperature distribution generated by pulsed wave laser in laser assisted micro milling (LAMM) is crucial. The selection of processing parameter by minimize the effect on the processing characteristic is decisive to ensure the machining quality is high. Determining the effect of heat generated in underneath surface is important to make sure that the cutting tools are able to cut the material with maximum depth of cut and minimum defects in term of tool wear and tool life. In this study the simulation was carried by using Ansys APDL. In order to confirm the actual and distribution irradiation of temperature from simulation, an experimental was done to validate the results. The experiment was conducted by using Nd:YAG laser with wavelength $1064 \mathrm{~nm}$.
\end{abstract}

Keyword: Finite element analysis; Laser assisted micro milling; Nd:YAG laser 